TECHNICAL NOTE

\author{
B.P. Liu \\ A. Aghaei Lasboo \\ M. Rozenfeld \\ T.A. Hijaz \\ S.F. Futterer \\ M.T. Walker
}

\section{Percutaneous Transosseous Translaminar Approach for Thecal Sac Access in Advanced Ankylosing Spondylitis with Instrumented Posterior Spinal Fusion}

\begin{abstract}
SUMMARY: A novel transosseous approach for percutaneous access of the lumbar subarachnoid space is described in a patient with advanced ankylosing spondylitis (AS) and instrumented spinal fusion who presented for myelography. Use of a coaxial threaded bone biopsy system to provide transosseous access to the thecal sac, imaging findings, and outcome are discussed. This technique provided access to an otherwise inaccessible subarachnoid space and is an alternative approach in the setting of advanced AS or posterior spinal fusion.
\end{abstract}

A nkylosing spondylitis (AS) is a seronegative spondyloarthropathy that can affect the sacroiliac joints, vertebral bodies, intervertebral disks, spinal facet joints, costovertebral joints, costotransverse joints, and the paravertebral soft tissues, including tendon and ligament attachments. ${ }^{1}$ In the setting of AS, access to the subarachnoid space for myelography can be difficult or impossible by using conventional lumbar puncture techniques due to spinal ankylosis, associated softissue abnormalities, and prior spinal fusions. ${ }^{2} \mathrm{We}$ describe a novel controlled transosseous approach to access the lumbar subarachnoid space safely in a patient with complete spinal fusion from AS and prior surgical fusion by using the Bonopty Bone Biopsy System (Radi Medical Systems, Uppsala Sweden).

\section{Technical Report}

A 44-year-old man with advanced AS and prior posterior spinal fusion from the occiput to the pelvis presented with progressive myelopathy (Fig. 1). CT and MR imaging were attempted but were limited due to metal artifacts from the extensive spinal fusion hardware. There were findings of upper and midthoracic spinal cord syringohydromyelia on MR imaging, but the presence/absence of subarachnoid adhesions, which could be causative, could not be determined (Fig 2). A total spine myelogram was requested via the $\mathrm{C} 1-2$ approach.

Review of prior cervical imaging showed extensive ossification at the craniocervical junction and a diminutive dorsal CSF space at C1-2. The C1-2 approach was deemed unsafe. A decision was made to attempt myelography via a lumbar approach by using CT guidance. The preprocedural CT scan showed complete ankylosis across the facets joints and between the lamina in conjunction with prior surgical bone graft fusion material over the posterior elements, resulting in a confluent continuous osseous barrier. A classic interlaminar lumbar puncture with standard spinal needles was not possible due to the extent of ossification/fusion. A lumbar transforaminal approach was considered but dismissed due to anatomic considerations and patient

Received April 21, 2009; accepted April 25.

From Feinberg School of Medicine of Northwestern University, Department of Radiology, Chicago, Illinois.

Please address correspondence to Matthew T. Walker, MD, Department of Radiology, Northwestern University, 676 North St. Clair St, Suite 1400, Chicago, IL 60611; e-mail: MWalker@nmff.org

DOI 10.3174/ajnr.A1701 positioning/comfort issues. It was decided to attempt access by drilling through the lamina and coaxially introducing a spinal needle into the thecal sac.

The patient was placed prone on the CT table and made comfortable, which required significant bolstering due to his fixed spinal kyphosis. After initial scanning of the lumbar spine, a left paramedian approach at L2-3 was selected due to the relative thinness of the osseous fusion in that position. Through a small incision, a 14-gauge 9.5-cm Bonopty penetration cannula and stylet (Radi Medical Systems) were advanced toward the target lamina. Upon reaching the bony surface, the stylet was exchanged for the 15-gauge 12.2 -cm drill stylet. Once the drill bit was anchored within the bone, a scan was obtained to confirm position. The drill was then slowly advanced, with periodic CT imaging, through the bone and the ossified ligamentum flavum until the dorsal epidural space was accessed. The penetration cannula was advanced over the tip of the drill into the dorsal epidural space, the drill was exchanged for a 22-gauge 15-cm Quincke spinal needle (Avid Medical, Toano, Virginia), and the thecal sac was accessed (Fig $3 A,-B$ ). CSF was obtained, and approximately $12 \mathrm{~mL}$ of iohexol (Omnipaque-240; Nycomed, Princeton, New Jersey) was injected. The patient was transferred to his stretcher and manipulated to allow the contrast to move throughout the spinal subarachnoid space (SAS). A CT scan of the entire spine was then obtained.

CT myelography revealed multiple complex subarachnoid adhesions with compartmentalization of the SAS in the lower thoracic region and cord compression. A complete block of CSF flow was identified at T8 (Fig 3C). The patient was taken to surgery for T5-T8 laminectomy/decompression, lysis of subarachnoid adhesions, and myelotomy for decompression of the spinal cord syringohydromyelia. At surgery, the initial durotomy exposed a SAS devoid of visible pulsatile CSF flow and a complex web of subarachnoid adhesions. These adhesions were carefully taken down, which re-established pulsatile CSF flow. Two separate myelotomies were performed through the dorsal cord of the thoracic spinal cord for decompression of the syringohydromyelia. A postoperative myelography was requested to assess the extent of the lysis, which was performed by using the same method as the preoperative myelography, and showed some decreased compartmentalization of the SAS. On clinical follow-up, the patient had improved symptomatically.

\section{Discussion}

AS is a seronegative spondyloarthropathy that results in progressive fusion of the sacroiliac joints and axial skeleton. ${ }^{1,3}$ AS 


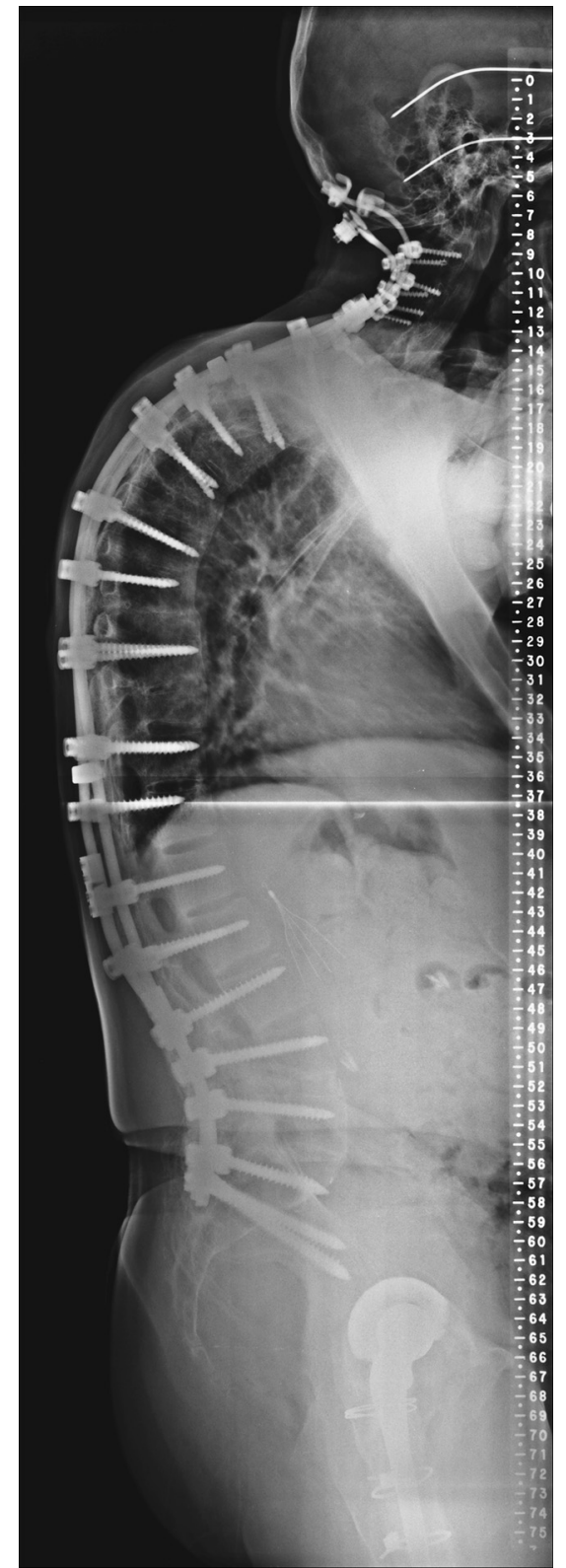

Fig 1. Lateral projection scoliosis radiograph demonstrates instrumented posterior spinal fusion from the occiput to the pelvis. There is an accentuated upper thoracic kyphosis and positive sagittal imbalance.

is an inflammatory process characterized by enthesitis and synovitis, leading to ossification of ligamentous structures such as the annulus fibrosis and ankylosis of the joints, including the apophyseal joints. The disease manifests clinically with pain and decreased range of motion of the spine. As the disease progresses, the entire spine may fuse together as 1 segment. Patients with AS often require posterior spinal fusion to stabilize or correct the spinal deformity. ${ }^{4}$ In advanced disease, extensive ossification can create a significant barrier to accessing the SAS. Any surgical instrumented fusion only adds to the complexity of access. When the spinal canal and SAS require evaluation and MR imaging is not diagnostic, CT myelography can play a role.

Jobanputra et $\mathrm{al}^{5}$ reported on a patient with AS and diskovertebral destruction who presented with signs of spinal cord compression. A noncontrast CT scan showed no evidence of

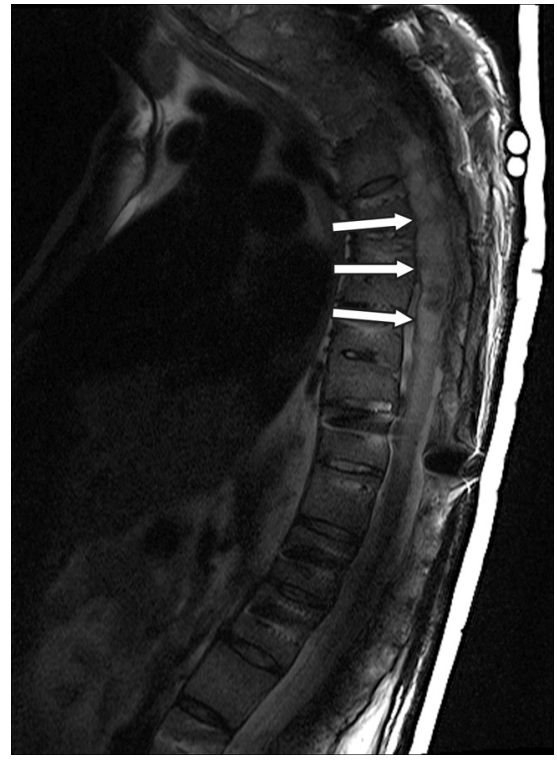

Fig 2. Sagittal fast spin-echo T2-weighted image of the thoracic spine demonstrates syringohydromyelia of the thoracic spinal cord with expansion of the cord (arrows). The syrinx extends from T2-T11. Portions of the spinal canal are obscured by artifacts.

cord compression, whereas CT myelography confirmed obstruction by an epidural process, which was histologically shown to represent bone with trabecula interspersed by fibrous granulation tissue and inflammatory cell infiltrate. The authors rightly concluded that $\mathrm{CT}$ alone was not a satisfactory method to study these lesions and that emphasis should be placed on contrast studies such as CT myelography or MR imaging. Despite advances in MR imaging technology, there remain many circumstances in which older technology such as CT myelography can be beneficial. Although the pathology in our patient was subarachnoid adhesions and not an epidural process, the same nondiagnostic results from noncontrast CT and artifacts-distorted MR imaging created a clinical dilemma: How can we safely access the thecal sac for a diagnostic myelography in a patient with complete spinal ankylosis?

The coaxial system we used differs from other bone biopsy systems in that it was designed with a drill stylet. In normal or osteoporotic bone, the 15-gauge drill stylet can be advanced at exactly the spiral thread profile of the drill with each turn. This allows controlled advancement through the bone under manual pressure. In well-mineralized or sclerotic bone, the drill stylet does not advance at the rate of the spiral thread profile but can cut through the bone with only manual pressure, though more turns and pressure are required to advance through the bone to the target. Controlled advancement of the coaxial system into the dorsal epidural space is preferable for subsequent safe delivery of the spinal needle into the thecal sac.

The use of the Bonopty Bone Biopsy System for controlled access of bony lesions has been described, but its use for transosseous thecal sac access has not. ${ }^{6}$ It is easy to use, and, in 1 study, was rated among the easiest to use when compared with 8 commonly used commercially available bone biopsy needles. ${ }^{7}$ This technique is useful in the spine and other osseous locations when a fine, precise, controlled transosseous needle advancement is desired. Although this technique was used for lumbar puncture and myelography in the setting of AS and instrumented spinal fusion, it has other potential uses, includ- 

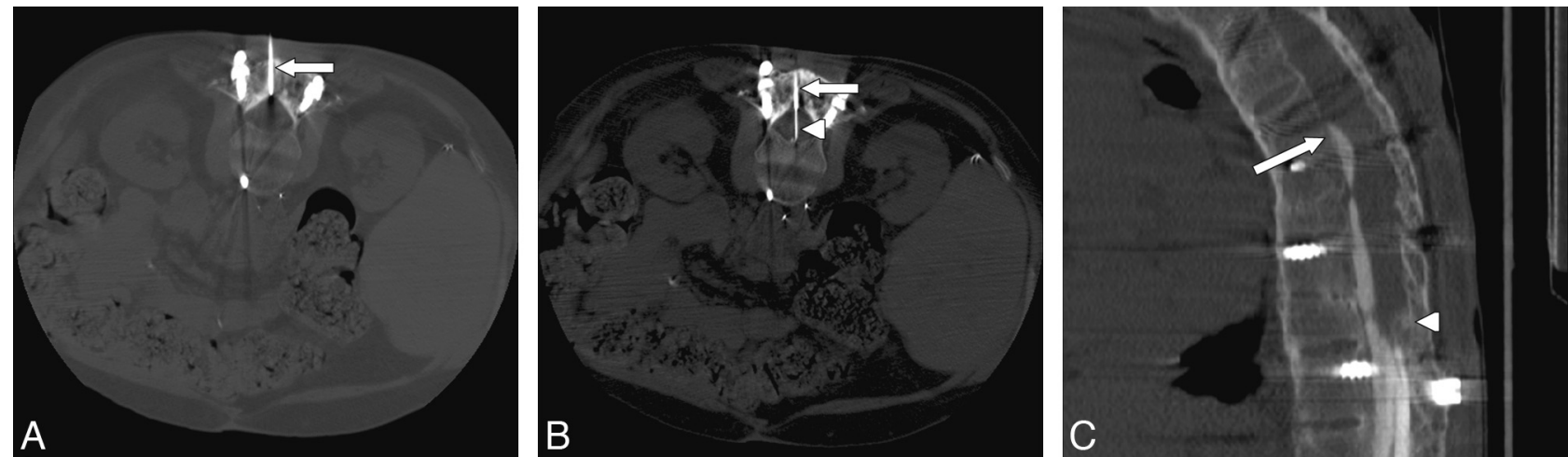

Fig 3. A, Axial CT scan through the $\mathrm{L} 2-3$ level with the patient prone shows the cannula (arrow) traversing a 2.5-cm thickness of bone graft, lamina, and ossified ligamentum flavum. $B$, Axial CT scan demonstrates placement of the 22-gauge spinal needle (arrowhead) through the cannula (arrow) and into the SAS. The needle tip was ventral, and though we did not puncture the ventral dura, that is a theoretic risk. In future attempts, we plan to measure the distance to the middle of the thecal sac and use a depth gauge or collar to identify our intended depth. We will then secure that depth with a clamp on the spinal needle so that it cannot inadvertently advance with gravity. $C$, Sagittal reconstruction of the CT myelogram demonstrates subarachnoid compartmentalization, adhesions, and a compete myelographic block ventrally at T8 (arrow) and dorsally at T12 (arrowhead).

ing controlled access to the epidural space for biopsies and direct synovial cyst punctures/fenestrations. At a minimum, this approach can be added to the interventional spine armamentarium for consideration in unique circumstances.

\section{References}

1. Hermann KG, Althoff $\mathrm{CE}$, Schneider U, et al. Spinal changes in patients with spondyloarthritis: comparison of MR imaging and radiographic appearances. Radiographics 2005;25:559-69, discussion 569-70

2. Bartleson JD, Cohen MD, Harrington TM, et al. Cauda equina syndrome secondary to long-standing ankylosing spondylitis. Ann Neurol 1983;14:662-69
3. Borenstein D. Arthritic disorders. In: Herkowitz HN, Garfin SR, Eismont FJ, et al, eds. The Spine. 5th ed. Philadelphia: Saunders; 2006:729-30

4. Hu SS, Ananthakrishnan D. Ankylosing spondylitis. In: Herkowitz HN, Garfin SR, Eismont FJ, et al, eds. The Spine. 5th ed. Philadelphia: Saunders: 2006:763-71

5. Jobanputra P, Kirkham B, Duke O, et al. Discovertebral destruction in ankylosing spondylitis complicated by spinal cord compression. Ann Rheum Dis 1988;47:344-47

6. DeFriend DE, Smith SP, Hughes PM. Percutaneous laser photocoagulation of osteoid osteomas under CT guidance. Clin Radiol 2003;58:222-26

7. Roberts CC, Morrison WB, Leslie KO, et al. Assessment of bone biopsy needles for sample size, specimen quality and ease of use. Skeletal Radiol 2005;34: 329-35 